\title{
Some Calculations in the Unstable Adams-Novikov Spectral Sequence ${ }^{1)}$
}

\author{
By \\ Martin BENDERSKY*
}

\section{§1. Introduction}

The unstable Adams-Novikov spectral sequence for a space $X$ is a sequence of groups $\left\{E_{r}(X)\right\}, r=2,3, \ldots$, which converge to the homotopy groups of $X$, and whose $E_{2}$-term depends on the complex cobordism groups of $X$. We investigate this spectral sequence when $X$ is the infinite special unitary group $S U$, or one of the finite groups $S U(n)$, or when $X$ is an odd sphere $S^{2 n+1}$.

The reader is referred to [2] for the construction and properties of the unstable Adams-Novikov spectral sequence. For some purposes, it is convenient to localize at a prime $p$, in which case the complex cobordism homology theory, based on the spectrum $M U$, is replaced by Brown-Peterson homology, based on the spectrum $B P$. We then have a useful spectral sequence with many of the properties of the stable Adams-Novikov spectral sequence. Namely, the filtrations are less than or equal to the filtrations in the unstable Adams spectral sequence based on mod- $p$ homology. When $X$ is a space for which $H_{*}(X ; B P)$ is free over the coefficient ring $\pi_{*}(B P)$ and cofree as a coalgebra, then the $E_{2}$-term is isomorphic to an Ext group in an abelian category (see $\S 2$; also $[2, \S 7])$. Furthermore, this Ext group may be computed as the homology of an unstable cobar complex which we describe explicitly in Section 2 . In particular, these considerations apply to the cases $X=S U, X=S U(n)$, or $X=S^{2 n+1}$.

We first consider the situation where $X$ is a $p$-local $H$-space with torsion-free homotopy and torsion-free homology. The results of Wilson [10] and the

Communicated by N. Shimada, November 21, 1978.

* Department of Mathematics, University of Washington, Seattle, Washington, 98195, U.S.A.

Present address: Department of Mathematics, Rider College, Lawrenceville, N. J. 08648 U.S.A.

1) Research in part supported by NSF Grant MCS 76-0823. 
general properties of the spectral sequence imply that the spectral sequence collapses, with $E_{2}^{0, *}(X) \approx \pi_{*}(X)$. In this case, the homotopy groups of $X$ (and even the homotopy type of $X$ ) are determined by the module of primitives $P H_{*}(X ; B P)$ as an unstable $\Gamma$-comodule (see $\S 3$ ). In particular, this is true for $X=S U$. We also give an explicit description of the generators of $E_{2}^{0, *}(S U)$.

In Section 4, we consider the case $X=S U(n)$. We compute $E_{2}^{1}, *(S U(n))$, which gives information about the pullback of the groups $\pi_{2 k}(S U(k))$ to $\pi_{2 k}(S U(n))$ for $n<k$. We also compute the kernel of the map $E_{2}^{2}, *(S U(n)) \rightarrow$ $E_{2}^{2}, *(S U(n+1))$. In the case of an odd prime $p$, the non-zero elements in this kernel survive to $E_{\infty}^{2, *}(S U(n))$ to give elements in homotopy related to the unstable image of the $J$-homomorphism. There is a similar but more complicated result for $p=2$.

In Section 5, we give a vanishing line for $E_{2}^{s, t}\left(S^{2 n+1}\right)$, and also for $E_{2}^{s, t}(S U(n))$. We then show that in a range of dimensions, $\pi_{*}\left(S^{2 n+1}\right)$ and $\pi_{*}(S U(n))$ may be computed as Ext groups in the category of unstable $\Gamma$-comodules. These calculations agree with, and extend those of Zabrodsky [11], and Mimura, Nishida and Toda [7].

Throughout the paper, space means simply connected Hausdorff topological space with basepoint, and map means continuous function preserving basepoint. The homotopy relation for spaces and maps is $\simeq$. The smash product is denoted by $\wedge$. In an algebraic situation, homomorphism means that the algebraic structure is preserved, and $\approx$ means a homomorphism which is an isomorphism onto. The ring of integers is denoted by $\mathbb{Z}$, and the rational numbers by $\boldsymbol{Q}$. For a prime number $p$, the ring of integers localized at $p$ is $\mathbb{Z}_{(p)}$. Except for 4.10 (3) all spaces are assumed to be $p$-localized. For an integer $n$, the ring of integers modulo $n$ is $\mathbb{Z}_{n}$.

\section{Acknowledgement}

The author wishes to acknowledge the contribution of E. B. Curtis to the final form of this paper.

\section{§2. The Unstable Adams-Novikov Spectral Sequence}

In this section, we summarize the main results of [2], which gives the construction and main properties of the unstable Adams-Novikov spectral 
sequence. When a prime $p$ is fixed, $B P$ refers to the Brown-Peterson spectrum associated with $p$. For a space $X$, the (reduced) homology groups of $X$ with coefficients in $B P$ are denoted by $H_{*}(X ; B P)$. The coefficient ring $\pi_{*}(B P)$ is called $A$, and the ring of co-operations $\pi_{*}(B P \wedge B P)$ is called $\Gamma$ ( $\Gamma$ is the $B P$ analogue of the dual of the Steenrod algebra).

The spectrum $B P$ defines a functor $B P(\cdot)$ from spaces to spaces by

$$
B P(X)=\lim _{n \rightarrow \infty} \Omega^{n}\left(B P_{n} \wedge X\right) .
$$

The unit in $B P$ is a map $i: S \rightarrow B P$ of the sphere spectrum $S$ to $B P$, which gives a map

$$
\eta=\eta(X): X \rightarrow B P(X)
$$

This gives rise to functors $D^{s}(\cdot)$ and a tower of fibrations

$$
\cdots \rightarrow D^{s}(X) \stackrel{\delta^{s}}{\longrightarrow} D^{s^{-1}}(X) \rightarrow \cdots \rightarrow D^{1}(X) \stackrel{\delta^{1}}{\longrightarrow} D^{0}(X)=X
$$

as follows. Inductively on $s$,

$$
\delta^{s}: D^{s}(X) \rightarrow D^{s-1}(X)
$$

is the fibration over $D^{s-1}(X)$ induced from the path-space fibration over $D^{s-1}(B P(X))$ by the map $D^{s-1}(\eta)$. The homotopy exact couple of this tower is called the unstable Adams spectral sequence for $X$ with respect to $B P$ and its terms are denoted $\left\{E_{r}^{s, t}(X ; B P)\right\}$. When the ring spectrum $B P$ is assumed, we call this the spectral sequence for $X$, and write $\left\{E_{r}^{s, t}(X)\right\}$. From $[2, \S 7]$, we have the following.

Theorem 2.2. For each simply-connected CW-space $X$, the spectral sequence $\left\{E_{r}^{s, t}(X ; B P)\right\}$ converges to the homotopy groups of $X$ localized at $p$. If $H_{*}(X ; B P)$ is free as an A-module, and cofree as a coalgebra, then

$$
E_{2}^{s, t}(X ; B P) \approx \operatorname{Ext}_{\mathscr{L}}^{s}\left(A[t], P H_{*}(X ; B P)\right) .
$$

Here $\mathscr{U}$ is the category of unstable $\Gamma$-comodules (which will be described below). $A[t]$ stands for the free A-module on one generator of degree $t$; $P H_{*}(X ; B P)$ stands for the submodule of primitives in $H_{*}(X ; B P)$. Furthermore, these Ext groups may be calculated as the homology of an unstable cobar complex $C^{*}, *\left(P H_{*}(X ; B P)\right)$, which will be described explicitly below.

We next recall some facts about $B P$ from [1] and [6]. First

$$
H_{*}\left(B P ; \mathbb{Z}_{(p)}\right) \approx \mathbb{Z}_{(p)}\left[m_{1}, m_{2}, \ldots\right]
$$




$$
H_{*}\left(B P \wedge B P ; Z_{(p)}\right) \approx H_{*}(B P)\left[t_{1}, t_{2}, \ldots\right]
$$

where degree $\left(m_{i}\right)=2\left(p^{i}-1\right)=$ degree $\left(t_{i}\right)$. The elements $t_{i}$ are chosen so that

$$
\eta_{R}\left(m_{k}\right)=\sum_{i=0}^{k} m_{i} t_{k-i}^{p^{i}}
$$

where $\eta_{R}$ is the right unit map. The elements $m_{0}$ and $t_{0}$ are to be interpreted as 1 . Then the Hazewinkel elements $v_{i}$ are defined recursively by the formula:

$$
v_{i}=p m_{i}-\sum_{j=1}^{i-1} m_{j} v_{i-j}^{p^{j}} .
$$

It is shown in [5] that the $v_{i}$ are in the image of the Hurewicz homomorphism $\pi_{*}(B P) \rightarrow H_{*}\left(B P ; \mathbb{Z}_{(p)}\right)$, which is a monomorphism, so the $v_{i}$ may be considered to be in $\pi_{*}(B P)$ also. Then

$$
\begin{aligned}
& A=\pi_{*}(B P) \approx Z_{(p)}\left[v_{1}, v_{2}, \ldots\right] \\
& \Gamma=\pi_{*}(B P \wedge B P) \approx A\left[t_{1}, t_{2}, \ldots\right] .
\end{aligned}
$$

The structure maps for $(A, \Gamma)$ consists of a product $\phi: \Gamma \otimes_{A} \Gamma \rightarrow \Gamma$, left and right unit maps $\eta_{L}, \eta_{R}: A \rightarrow \Gamma$, a counit map $\varepsilon: \Gamma \rightarrow A$, and a diagonal map $\psi$ : $\Gamma \rightarrow \Gamma \otimes_{A} \Gamma$. The product $\phi$ and the left unit map $\eta_{L}$ are built into the description of $\Gamma$ as a polynomial algebra over $A$. The right unit map $\eta_{R}$ is given above (2.3) for the $m_{i}$, and thereby, using (2.4), for the $v_{i}$ also. The diagonal map $\psi$ satisfies the formula

$$
\sum_{i+j=n} m_{i} \psi\left(t_{j}\right)^{p^{2}}=\sum_{i+j+k=n} m_{i} t_{j}^{p^{i}} \otimes t_{k}^{p^{i+j}}
$$

The notation $M \otimes_{A} N$ requires that $M$ be a right $A$-module, and that $N$ be a left $A$-module. $\quad \Gamma$ is a right $A$-module by $\eta_{R}$, and a left $A$-module by $\eta_{L}$. The notation $\eta_{L}$ is usually suppressed, and $\eta_{R}$ is sometimes called $\eta$.

There is a formal group law associated with $B P$ as follows. Let $\boldsymbol{C} P^{\infty}$ stand for infinite dimensional complex projective space, and let

$$
\mu: \boldsymbol{C} P^{\infty} \times \boldsymbol{C} P^{\infty} \rightarrow \boldsymbol{C} P^{\infty}
$$

be the map which classifies the tensor product of the canonical line bundles. Then (in unreduced homology),

$$
\begin{aligned}
& H^{*}\left(\boldsymbol{C} P^{\infty} ; B P\right) \approx A \llbracket X \rrbracket \\
& H^{*}\left(\boldsymbol{C} P^{\infty} \times \boldsymbol{C} P^{\infty} ; B P\right) \approx A \llbracket X_{1}, X_{2} \rrbracket,
\end{aligned}
$$

which are respectively the formal power series rings in one and two variables over $A$. The formal group law $F$ is defined by the formal power series: 


$$
F\left(X_{1}, X_{2}\right)=\mu^{*}(X)=\sum a_{i, j} X_{1}^{i} X_{2}^{j}
$$

where the coefficients $a_{i, j}$ belong to $A_{2 i+2 j-2}$. As in [1], let log be the formal power series (over $A \otimes Q$ ):

$$
\log (X)=\sum_{i \geqq 0} m_{i} X^{p^{i}}
$$

Let exp be the formal power series inverse to $\log$, which satisfies $\log (\exp X)$ $=X$. Then

$$
F\left(X_{1}, X_{2}\right)=\exp \left(\log X_{1}+\log X_{2}\right) .
$$

The formal group law $F$ is associative and commutative. Elements $z_{i}$ from $\Gamma$ may be substituted for the undeterminates, and we write $\sum^{F} z_{i}$ for $F\left(z_{1}, F\left(z_{2}\right.\right.$, ...)). Then formula (2.5) becomes

$$
\sum_{j}^{F} \psi\left(t_{j}\right)=\sum_{j, k}^{F} t_{j} \otimes t_{k}^{p^{j}}
$$

There is a canonical anti-automorphism $c: \Gamma \rightarrow \Gamma$, which satisfies $c \eta_{L}=\eta_{R}$ and $c \eta_{R}=\eta_{L}$. This gives a formal group law $F^{*}$ conjugate to $F$, defined by the formula:

$$
\sum^{F^{*}} z_{i}=c\left(\sum^{F} c\left(z_{i}\right)\right)
$$

Notation 2.9. Let $c\left(t_{i}\right)=h_{i}$.

The elements $h_{i}$ satisfy the following formulas, which are obtained by applying $c$ to (2.3), (2.5), and (2.12):

$$
\begin{aligned}
& m_{n}=\sum_{i=0}^{n}\left(h_{n-i}\right)^{p^{i}} \eta\left(m_{i}\right) \\
& \sum_{i+j=n} \psi\left(h_{j}\right)^{p^{i}} \eta\left(m_{i}\right)=\sum_{i+j+k=n} h_{k}^{p^{i+j}} \otimes h_{j}^{p^{i}} \eta\left(m_{i}\right) \\
& \sum^{F^{*}} \psi\left(h_{j}\right)=\sum^{F^{*}} h_{k}^{p^{j}} \otimes h_{j} .
\end{aligned}
$$

We also have $\Gamma \approx A\left[h_{1}, h_{2}, \ldots\right]$.

For each finite sequence of non-negative integers, $I=\left(i_{1}, i_{2}, \ldots, i_{n}\right)$, let

$$
h^{I}=h_{1}^{i_{1}} h_{2}^{i_{2}} \cdots h_{n}^{i_{n}} \text {. }
$$

The length of $I$ is the integer $l(I)=i_{1}+\cdots+i_{n}$.

Definition 2.13. For each non-negatively graded, free left $A$-module $M$, let $U(M)$ be the sub- $A$-module of $\Gamma \otimes_{A} M$ spanned by all elements of the form $h^{I} \otimes_{A} m$ where $2 l(I)<$ degree $(m)$.

For an arbitrary non-negatively graded left $A$-module let 


$$
F_{1} \stackrel{f}{\longrightarrow} F_{0} \rightarrow M \rightarrow 0
$$

be exact with $F_{0}$ and $F_{1}$ free. Then define

$$
U(M)=\operatorname{coker}\left(U(f): U\left(F_{1}\right) \rightarrow U\left(F_{0}\right)\right) .
$$

It is easily verified that $U(M)$ is independent of $F_{1}, F_{0}$ and $f$.

Remark 2.14. In $[2,(7.4)]$, the functor $U(\cdot)$ is defined in terms of another functor $G(\cdot)$ (specifically, $U(M)$ is the submodule of primitives in $G(M)$ ). The discussion of $[2,(8.7)]$ shows that the two definitions of $U(M)$ agree.

There is a $\Gamma$-comodule structure on $\Gamma \otimes_{A} M$ by the map

$$
\psi \otimes 1: \Gamma \otimes_{A} M \rightarrow \Gamma \otimes_{A} \Gamma \otimes_{A} M .
$$

An easy induction using (2.12) shows that $\psi \otimes 1$ takes $U(M)$ to $U^{2}(M)$, and hence induces a map

$$
\delta^{U}: U(M) \rightarrow U^{2}(M)
$$

There is also a counit map $\varepsilon^{U}: U(M) \rightarrow M$ induced by the counit map in $\Gamma$. In the notation of $[2, \S 5],\left(U, \delta^{U}, \varepsilon^{U}\right)$ is a cotriple on the category $\mathscr{A}$ of nonnegatively graded left $A$-modules.

A module $M$ in $\mathscr{A}$ with a $U$-structure will be called an unstable $\Gamma$-comodule (see $[2,(7.4)])$. This means that there is a map $\psi: M \rightarrow U(M)$ such that the following diagrams commuie:
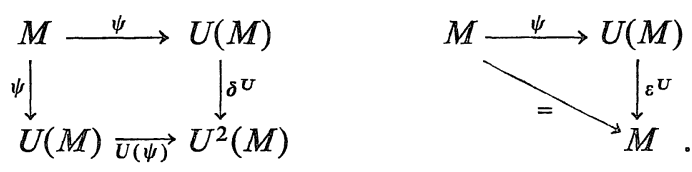

The category of unstable $\Gamma$-comodules will be called $\mathscr{U}(\mathscr{U}$ is called $\mathscr{A}(U)$ in [2]). By construction $\mathscr{U}$ is an abelian category. To simplify notation, we shall write

$$
\operatorname{Ext}_{\mathscr{L}}^{s, t}(M) \text { for } \operatorname{Ext}_{\mathscr{L}}^{s}(A[t], M)
$$

for $M$ in $\mathscr{U}$. These Ext groups may be calculated as the homology groups of an unstable cobar complex $C^{*}, *(M)$. Specifically, for each pair $(s, t)$ of nonnegative integers,

$$
C^{s, t}(M)=U^{s}(M)_{t} .
$$

As is customary, we write $\left[\gamma_{1}|\cdots| \gamma_{s}\right] m$ for the element $\gamma_{1} \otimes \cdots \otimes \gamma_{s} \otimes m$ in $U^{s}(M)$. The complementary degree $t$ of such an element is the integer 


$$
t=\operatorname{deg}\left(\gamma_{1}\right)+\cdots+\operatorname{deg}\left(\gamma_{s}\right)+\operatorname{deg}(m)
$$

The differential

$$
d^{s}: C^{s, t}(M) \rightarrow C^{s+1, t}(M)
$$

is given by

$$
\begin{aligned}
d^{s}\left[\gamma_{1}|\cdots| \gamma_{s}\right] m= & {\left[1\left|\gamma_{1}\right| \cdots \mid \gamma_{s}\right] m } \\
& +\sum(-1)^{j}\left[\gamma_{1}|\cdots| \gamma_{j}^{\prime}\left|\gamma_{j}^{\prime \prime}\right| \cdots \mid \gamma_{s}\right] m \\
& +\sum(-1)^{s+1}\left[\gamma_{1}|\cdots| \gamma_{s} \mid \gamma^{\prime}\right] m^{\prime \prime}
\end{aligned}
$$

where $\psi\left(\gamma_{j}\right)=\sum \gamma_{j}^{\prime} \otimes \gamma_{j}^{\prime \prime}$ and $\psi(m)=\sum \gamma^{\prime} \otimes m^{\prime \prime}$. Then, from [2, (9.3)], we have

$$
\operatorname{Ext}_{\mathscr{L}}^{s, t}(M) \approx H^{s, t}\left(C^{*, *}(M)\right) \text {. }
$$

Remark 2.16. As in the stable case, there is a smaller "normalized" cobar complex $\widetilde{C}^{*, *}(M)$. This is obtained from the functor

$$
\tilde{U}(M)=\operatorname{ker}\left\{\varepsilon^{U}: U(M) \rightarrow M\right\}
$$

by

$$
\widetilde{C}^{s, *}(M)=\tilde{U}^{s}(M) .
$$

The inclusion $\tilde{C}(M) \rightarrow C(M)$ is a chain equivalence. This gives the following "easy" vanishing line.

Proposition 2.17. If $M$ is a $k$-connected unstable $\Gamma$-comodule, then $\operatorname{Ext}_{\mathscr{\mathscr { U }}}^{s, t}(M)=0$, for $t \leqq 2(p-1) s+k$.

The proof is immediate because $\widetilde{C}^{s, t}(M)=0$ for $t \leqq 2(p-1) s+k$.

This vanishing line will be improved in Section 5 .

\section{§3. The Spectral Sequence for $\mathbb{S} \mathbb{U}$}

When the space $X$ is $S U$ (=the infinite special unitary group), the unstable Adams-Novikov spectral sequence simplifies considerably, as shown below; compare with the unstable spectral sequence for $S U$ based on $\bmod p$ homology in $[4$, p. 191]. In fact, we have the following general result.

Theorem 3.1. Let $X$ be an $H$-space with torsion-free homology and torsion-free homotopy. Then the unstable Adams-Novikov spectral sequence for $X$ collapses. That is, 


$$
E_{2}^{s, t}(X) \approx\left\{\begin{array}{lll}
\pi_{t}(X), & \text { for } & s=0 \\
0, & \text { for } & s>0
\end{array}\right.
$$

Before giving the proof of Theorem 3.1, we recall the results of Wilson [10] which are the main ingredients of the proof.

Wilson's Spaces $Y_{k}$ (3.2). There are indecomposable $H$-spaces $Y_{k}(k=1,2$, ...), which have the following properties.

(i) $Y_{k}$ is $(k-1)$-connected, and $\pi_{k}\left(Y_{k}\right)=Z_{(p)}$.

(ii) For $k \neq 2\left(p^{n}+p^{n-1}+\cdots+1\right), \Omega Y_{k+1} \simeq Y_{k}$. For $k=2\left(p^{n}+p^{n-1}+\cdots\right.$ +1), $\Omega Y_{k+1} \simeq Y_{k} \times Y_{p k}$.

(iii) If $f: Y_{k} \rightarrow Y_{k}$ is a continuous map which induces an isomorphism $f_{*}$ : $\pi_{k}\left(Y_{k}\right) \approx \pi_{k}\left(Y_{k}\right)$, then $f$ is a homotopy equivalence.

(iv) If $X$ is a $H$-space with torsion-free homology and torsion-free homotopy, then $X$ is a product of the $Y_{k}$ 's:

$$
X \simeq \prod_{\alpha} Y_{k_{\alpha}}
$$

(but not necessarily as $H$-spaces).

(v) Let $B P_{k}$ be the $k$-th space in the $\Omega$-spectrum for $B P$. Then there are maps $i$ and $j$

$$
Y_{k} \stackrel{i}{\longrightarrow} B P_{k} \stackrel{j}{\longrightarrow} Y_{k}
$$

where $j \circ i$ is a homotopy equivalence. Moreover we may consider $B P_{k}=Y_{k} \times Z$ where $Z$ is a space at least $k$-connected.

Proof of Theorem 3.1. For each of the spaces $Y_{k}$, consider the following homotopy-commutative diagram:

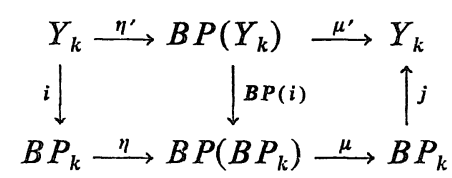

where $\eta^{\prime}=\eta\left(Y_{k}\right), \eta=\eta\left(B P_{k}\right)$; the map $\mu$ is induced by the product in $B P$, and $\mu^{\prime}=j \circ \mu \circ B P(i)$. The properties of $B P$ as a ring spectrum imply that $\mu \circ \eta$ is a homotopy equivalence. Hence $\mu^{\prime} \circ \eta^{\prime}$ induces $\pi_{k}\left(Y_{k}\right) \approx \pi_{k}\left(Y_{k}\right)$, and so by (3.2, (iii)) must be a homotopy equivalence. By the construction (2.1) or [2, (2.4)] this implies that the spectral sequence for $Y_{k}$ collapses. It follows that for any product of the $Y_{k}$ 's, the spectral sequence collapses. By (3.2, (iv)), $X$ is such a space, so the spectral sequence collapses for $X$.

Q.E.D. 
Remark 3.3. Using the facts that $S U$ is an $H$-space with torsion-free homology and torsion-free homotopy, we see that the spectral sequence collapses for $S U$. For an alternative approach, see Remark 3.8.

We proceed to analyze the image of the $B P_{*}$-Hurewicz homomorphism

$$
\begin{aligned}
\operatorname{im}\left\{\pi_{*}(S U) \rightarrow H_{*}(S U ; B P)\right\} & \approx E_{2}^{0, *}(S U) \\
& \approx \operatorname{Ext}_{\mathscr{\ell}}^{0, *}\left(P H_{*}(S U ; B P)\right) .
\end{aligned}
$$

Here $P H_{*}(S U ; B P)$ stands for the submodule of primitives in $H_{*}(S U ; B P)$ considered as an unstable $\Gamma$-comodule. For later use, we give a description of $P H_{*}(S U ; B P)$. Recall from [8] that there is a map

$$
f: S^{1} \wedge C P^{\infty} \rightarrow S U \text {. }
$$

As in [1], let $\beta_{k}$, for $k=1,2, \ldots$ be the natural generator of $H_{2 k}\left(\mathbb{C} P^{\infty} ; B P\right)$. Let $f_{*}\left(\iota_{1} \wedge \beta_{k}\right)=x_{2 k+1}$, which is a primitive element in $H_{2 k+1}(S U ; B P) . \quad H_{*}(S U$; $B P)$ is cofree as a coalgebra, and $P H_{*}(S U ; B P)$ is the free $A$-module generated by $\left\{x_{2 k+1}\right\}, k=1,2, \ldots$.

To describe the unstable $\Gamma$-coaction on $\mathrm{PH}_{*}(S U ; B P)$ we proceed as follows. As in [1], by abuse of notation, let $\beta_{k}$ also stand for the generator of $H_{2 k}\left(\mathbf{C P} P^{\infty}\right.$; $M U)$, and let $b_{k}^{M U}$ be the generator of $H_{2 k}(M U ; M U)$. Then from [1, (11.4)] the formula for the coaction in $H_{*}\left(\mathbb{C} P^{\infty} ; M U\right)$ is

$$
\psi\left(\beta_{k}\right)=\sum_{j}\left(\sum_{s} b_{s}^{M U}\right)_{k-j}^{j} \otimes \beta_{j}
$$

where $\left(\sum_{s} b_{s}^{M U}\right)_{k-j}^{j}$ stands for the terms of degree $2 k-2 j$ in $\left(\sum_{s} b_{s}^{M U}\right)^{j}$. The Quillen idempotent induces a map from $H_{*}(M U ; M U)$ to $H_{*}(B P ; B P)$ which sends $\sum_{s} b_{s}^{M U}$ to $\sum_{s}^{F^{*}} h_{s}$, as shown in $[2,(8.3)]$. Thus the formula for the coaction in $H_{*}\left(\boldsymbol{C} P^{\infty} ; B P\right)$ is

$$
\psi\left(\beta_{k}\right)=\sum_{j}\left(\sum_{s}^{F^{*}} h_{s}\right)_{k-j}^{j} \otimes \beta_{j} .
$$

Then by naturality of $f_{*}$, the formula for the coaction in $H_{*}(S U ; B P)$ is

$$
\psi\left(x_{2 k+1}\right)=\sum_{j}\left(\sum_{s}^{F^{*}} h_{s}\right)_{k-j}^{j} \otimes x_{2 j+1} .
$$

It would be awkward to calculate the groups $\operatorname{Ext}_{\mathscr{Z}}^{0} *\left(P H_{*}(S U ; B P)\right)$ directly. Instead, we do the following. Define an $A$-linear map

$$
\phi: P H_{*}(S U ; B P) \rightarrow P H_{*+2}(S U ; B P)
$$

by 


$$
\phi\left(x_{2 i+1}\right)=\sum_{k=1}^{i+1} k a_{i-k+1} x_{2 k+1}
$$

where $a_{s}=a_{s, 1}$ (the $a_{i, j}$ are the $B P$-formal group law coefficients). The $a_{s}$ may be computed recursively by $a_{0}=1$, and the formula (see $[1,(10.1)]$ ):

$$
\sum_{n \geqq 0} p^{n} m_{n}=\left(\sum_{s \geqq 0} a_{s}\right)^{-1}
$$

Let $\phi^{k}$ stand for the $k$-th iterate of the map $\phi$. The following is motivated by Toda's proof of the Bott Periodicity Theorem [8].

Proposition 3.5. For each non-negative integer $k, \phi^{k}\left(x_{3}\right)$ generates $E_{2}^{0,2 k+3}(S U)$.

Proof. The Hopf construction applied to the map $\mu: C P^{\infty} \times C P^{\infty}$ gives a map:

$$
H(\mu): S^{1} \wedge C P^{\infty} \wedge C P^{\infty} \rightarrow S^{1} \wedge C P^{\infty} .
$$

The restriction of $H(\mu)$ to $S^{1} \wedge S^{2} \wedge C P^{\infty}$ will be called $\zeta$. $\zeta$ induces a map in homology:

$$
\zeta_{*}: H_{*}\left(\boldsymbol{C} P^{\infty} ; B P\right) \rightarrow H_{*+2}\left(\mathbb{C} P^{\infty} ; B P\right) .
$$

By dualizing the formal group law for $B P$, we see that

$$
\zeta_{*}\left(\beta_{i}\right)=\sum_{k=1}^{i+1} k a_{i-k+1} \beta_{i}
$$

Consider the composite map (which will be called $\zeta_{k+1}$ ):

$$
\begin{gathered}
S^{2 k+3} \stackrel{\text { inc1. }}{\longrightarrow} S^{2 k+1} \wedge C P^{\infty} \stackrel{S^{2 k+1}(\zeta)}{\longrightarrow} S^{2 k-1} \wedge C P^{\infty} \stackrel{S^{2 k-4}(\zeta)}{\longrightarrow} \\
\quad \cdots \stackrel{S^{2}(\zeta)}{\longrightarrow} S^{3} \wedge C P^{\infty} \stackrel{\zeta}{\longrightarrow} S^{1} \wedge C P^{\infty} \stackrel{f}{\longrightarrow} S U .
\end{gathered}
$$

The map $\zeta_{k+1}$ induces a map of the unstable Adams-Novikov spectral sequences

$$
\left(\zeta_{k+1}\right)_{*}: E_{2}^{0,2 k+3}\left(S^{2 k+3}\right) \rightarrow E_{2}^{0,2 k+3}(S U)
$$

with $\left(\zeta_{k+1}\right)_{*}\left(\iota_{2 k+3}\right)=\phi^{k}\left(x_{3}\right)$. This shows that $\phi^{k}\left(x_{3}\right)$ is a cycle. By examining the coefficient of $x_{2 m+1}$, where $m$ is the integer $k-1$ reduced modulo $p-1$, we see that $\phi^{k}\left(x_{3}\right)$ is not divisible by $p$. Therefore, $\phi^{k}\left(x_{3}\right)$ is a generator of $E_{2}^{0,2 k+3}(S U)$.

Q.E.D.

Remarks 3.6. (i) The above proof also shows that the composite map $\zeta_{k+1}$ is a generator of $\pi_{2 k+1}(S U)$. This reproves a theorem of Toda [8].

(ii) Some examples of the generators of $E_{2}^{0}, *(S U)$ for the prime $p=2$ are the following 


$$
\begin{aligned}
& \phi\left(x_{3}\right)=2 x_{5}-v_{1} x_{3} \\
& \phi^{2}\left(x_{3}\right)=6 x_{7}-6 v_{1} x_{5}+3 v_{1}^{2} x_{3} \\
& \phi^{3}\left(x_{3}\right)=24 x_{9}-36 v_{1} x_{7}+30 v_{1}^{2} x_{5}-\left(12 v_{2}+9 v_{1}^{2}\right) x_{3} .
\end{aligned}
$$

Theorem 3.7. Let $Y$ be an $H$-space with torsion-free homology and torsion-free homotopy. Suppose that $X$ is a space such that $H_{*}(X ; B P)$ is cofree as a coalgebra over $A$, and that

$$
P H_{*}(X ; B P) \approx P H_{*}(Y ; B P)
$$

as unstable $\Gamma$-comodules. Then $X \simeq Y$.

Proof. By (3.1), the unstable Adams-Novikov spectral sequence for $Y$ collapses. Both $X$ and $Y$ satisfy the assumptions of $(2.2)$, so $E_{2}^{*}, *(X) \approx E_{2}^{*}, *(Y)$. Thus the spectral sequence collapses for $X$ too, and $\pi_{*}(X)$ is free over $\mathbb{Z}_{(p)}$. Also, $H_{*}\left(X ; \mathbb{Z}_{(p)}\right)$ is free over $\mathbb{Z}_{(p)}$ because of the isomorphisms:

$$
\begin{aligned}
H_{*}\left(B P ; \mathbb{Z}_{(p)}\right) \otimes_{Z_{(p)}} H_{*}\left(X ; \mathbb{Z}_{(p)}\right) & \approx \pi_{*}\left(B P \wedge K\left(\mathbb{Z}_{(p)}\right) \wedge X\right) \\
& \approx H_{*}\left(B P ; \mathbb{Z}_{(p)}\right) \otimes_{A} H_{*}(X ; B P) .
\end{aligned}
$$

Therefore, by $[1,(5.23)], X$ is an $H$-space with torsion-free homotopy and torsion-free homology. By (3.2, (iv)), $X$ and $Y$ are each a product of the $Y_{k}$ 's. As $\pi_{*}(X) \approx \pi_{*}(Y)$, the factors which occur for $X$ are the same as those which occur for $Y$.

Q.E.D.

Remark 3.8. It is possible by a lengthy calculation to determine the coaction in the space $Y=Y_{3} \times Y_{5} \times \cdots \times Y_{2 p-1}$, and then to show that

$$
P H_{*}(S U ; B P) \approx P H_{*}(Y ; B P)
$$

as unstable $\Gamma$-comodules. Thus (3.7) implies that

$$
S U \simeq Y_{3} \times Y_{5} \times \cdots \times Y_{2 p-1} .
$$

Making use of $\left(3.2\right.$, (iv)), we have that $\Omega^{2} Y_{3} \simeq S^{1} \times Y_{2 p-1}$, and that for $2 \leqq j$ $\leqq p-1, \Omega^{2} Y_{2 j+1} \simeq Y_{2 j-1}$. Thus

$$
\begin{aligned}
\Omega^{2}(S U) & \simeq S^{1} \times Y_{2 p-1} \times Y_{3} \times \cdots \times Y_{2 p-3} \\
& \simeq S^{1} \times S U
\end{aligned}
$$

which is the (complex) Bott periodicity theorem.

\section{§4. Calculations of $\mathbb{E}_{2}^{1}, *(S U($ R $))$}

Let $S U(n)$ be the spectral unitary group in $n$ variables. $H_{*}(S U(n) ; B P)$ is 
free as an $A$-module, and cofree as a coalgebra. The submodule of primitives $\mathrm{PH}_{*}(\mathrm{SU}(n) ; B P)$ will be called $M(n)$. By $(2.2)$, we have

$$
E_{2}^{s, t}(S U(n)) \simeq \operatorname{Ext}_{\mathscr{\ell}}^{s, t}(M(n)) .
$$

As an $A$-module, $M(n)$ is freely generated by the elements $x_{3}, x_{5}, \ldots, x_{2 n-1}$ defined in Section 3. The unstable $\Gamma$-comodule structure $\psi: M(n) \rightarrow U M(n)$ is given by formula (3.4). The groups $\operatorname{Ext}_{\mathscr{Z}}^{s, t}(M(n))$ are the homology of the unstable cobar complex. By sparseness, we have

$$
E_{2}^{\mathrm{s}, t}(S U(n))=0, \quad \text { for } t \text { even } .
$$

Consider the fibration

$$
S U(n) \rightarrow S U(n+1) \rightarrow S^{2 n+1} .
$$

Passing to $B P_{*}$-homology, and then taking primitives, we obtain a short exact sequence of unstable $\Gamma$-comodules:

$$
0 \rightarrow M(n) \rightarrow M(n+1) \rightarrow A[2 n+1] \rightarrow 0 .
$$

This induces a long exact sequence of Ext groups, which, after identifying them as $E_{2}$-terms, becomes

$$
\cdots \rightarrow E_{2}^{s, t}(S U(n)) \rightarrow E_{2}^{s, t}(S U(n+1)) \rightarrow E_{2}^{s, t}\left(S^{2 n+1}\right) \stackrel{\delta}{\longrightarrow} \cdots
$$

where $\delta$ has bidegree $(1,0)$. The indexing $(s, t)$ is such that $t-s$ is the homotopy dimension, whereas in [2], as in the stable case (e.g. [6]), $t-s$ is stem dimension.

\section{Proposition 4.2.}

(i) $E_{2}^{s, t}(S U(n)) \approx E_{2}^{s, t}(S U(n+1))$, for $t<2 n+2 s$.

(ii) $E_{2}^{0, t}(S U(n)) \approx 0, \quad$ for $t \geqq 2 n$, $E_{2}^{0,2 i+1}(S U(n)) \approx Z_{(p)}, \quad$ for $1 \leqq i<n$.

(iii) $E_{2}^{1,2 n+1}(S U(n)) \approx \mathbb{Z}_{(p)} /(n !) \mathbb{Z}_{(p)}$.

(iv) For $n<k$, the inclusion $S U(n) \rightarrow S U(k)$ induces a monomorphism

$$
E_{2}^{1,2 k+1}(S U(n)) \rightarrow E_{2}^{1,2 k+1}(S U(k)) .
$$

Proof. Statements (i) and (ii) follow immediately from the long exact sequences (4.1), the easy vanishing line (2.17) for $E_{2}^{s, t}\left(S^{2 n+1}\right)$, and the calculation of $E_{2}^{0, *}(S U)$. Part (iii) follows from (3.5) and (4.1). Part (iv) follows from (4.1) and the fact that $E_{2}^{0, t}\left(S^{2 n+1}\right)=0$, for $t \neq 2 n+1$ Q.E.D.

The calculation of the rest of the 1-line for $S U(n)$ is the calculation of which elements of $E_{2}^{1,2 k+1}(S U(k))$ pull back to $E_{2}^{1}, 2 k+1(S U(n))$, for $n<k$. The 
difficulty in working directly with the cobar complex $C^{*, *}(M(n))$ is the formal sum which occurs in the expression for the differential. That is,

$$
\begin{aligned}
d\left(x_{2 k+1}\right) & =1 \otimes x_{2 k+1}-\psi\left(x_{2 k+1}\right) \\
& =-\sum_{j=1}^{k-1}\left(\sum_{s}^{F^{*}} h_{s}\right)_{k-j}^{j} \otimes x_{2 j+1} .
\end{aligned}
$$

To overcome this difficulty, we introduce a map $\bar{e}: \Gamma \rightarrow \mathbb{Q}$ as follows. From Section 2 , we see that $\Gamma \otimes_{\mathbf{Z}} \boldsymbol{Q}$ is a polynomial algebra over $\mathbb{Q}$ generated by the $m_{k}$ and the $\eta_{R}\left(m_{k}\right), k=1,2, \ldots$.

Definition 4.3. Let $\bar{e}: \Gamma \otimes_{z} \mathbb{Q} \rightarrow \mathbb{Q}$ be the homomorphism defined on the generators by

$$
\begin{aligned}
& \bar{e}\left(m_{k}\right)=1 / p^{k} \\
& \bar{e}\left(\eta_{R}\left(m_{k}\right)\right)=0
\end{aligned}
$$

for $k=1,2, \ldots$ Also, let $\bar{e}: \Gamma \rightarrow \mathbb{Q}$ denote the restriction of this map to $\Gamma$.

Lemma 4.4. For the Hazewinkel elements $v_{i}$ in $\Gamma$, we have $\bar{e}\left(v_{1}\right)=1$, $\bar{e}\left(v_{i}\right)=0$ for $i>1$.

Proof. $v_{1}=p m_{1}$, so $\bar{e}\left(v_{1}\right)=1$. For $i>1$, the $v_{i}$ are defined by formula (2.4), and it follows by induction on $i$ that $\bar{e}\left(v_{i}\right)=0$ for $i>1$.

Q.E.D.

In the unstable cobar complex $C^{*, *}\left(S^{2 n+1}\right)$, we have $C^{0, *}\left(S^{2 n+1}\right) \approx A$. For $a$ in $A, d(a)=\eta_{R}(a)-a$, so

$$
\bar{e}(d(a))=-\bar{e}(a) .
$$

Therefore, $\bar{e}(d(A)) \subset \mathbb{Z}_{(p)}$. Passing to the homology of the unstable cobar complex, we obtain a well-defined map

$$
e: E_{2}^{1}, *\left(S^{2 n+1}\right) \rightarrow \mathbb{Q} / \mathbb{Z}_{(p)}
$$

which is (up to sign) an unstable analogue of the complex Adams $e$-invariant localized at $p$. The description of $E_{2}^{1}, *\left(S^{2 n+1}\right)$ in $[2, \S 9]$ shows that $e$ is a monomorphism. Thus the order of any element $\alpha$ in $E_{2}^{1} * *\left(S^{2 n+1}\right)$ is the same as the order of $e(\alpha)$ in $\boldsymbol{Q} / \mathbb{Z}_{(p)}$.

Definition 4.5. For each pair $(k, j)$ of positive integers, we define a rational number $b_{k, j}$ as follows. For each $j \geqq 1$, let

$$
\left(Y+\frac{Y^{p}}{p}+\frac{Y^{p^{2}}}{p^{2}}+\cdots\right)^{j}=\sum_{k \geqq j} b_{k, j} Y^{k}
$$

in the formal power series ring $Q \llbracket Y \rrbracket$. 
The properties of $\bar{e}$ which will be used in the proof of (4.7) are the following. Recall from (2.9) that $h_{k}=c\left(t_{k}\right)$, where $c$ is the canonical anti-automorphism.

Lemma 4.6. (i) $\bar{e}\left(h_{k}\right)=1 / p^{k}$,

(ii) $\bar{e}\left(\sum^{F^{*}} h_{s}\right)_{k-j}^{j}=b_{k, j}$.

Proof. Apply the canonical anti-automorphism $c$ to (2.3) to obtain

$$
m_{k}=\eta_{R}\left(m_{k}\right)+h_{1}^{p k-1} \eta_{R}\left(m_{k-1}\right)+\cdots+h_{k} .
$$

As $\bar{e}$ is 0 on the image of $\eta_{R}$, part (i) follows. For part (ii), we have

$$
\begin{aligned}
\bar{e}\left(\sum^{F^{*}} h_{s}\right)_{k-j}^{j} & =\bar{e}\left(\sum h_{s}\right)_{k-j}^{j}=\left(1+\frac{Y^{p-1}}{p}+\frac{Y^{p^{2}-1}}{p^{2}}+\cdots\right)_{k-j}^{j} \\
& =\left(Y+\frac{Y^{p}}{p}+\frac{Y^{p^{2}}}{p^{2}}+\cdots\right)_{k}^{j} \\
& =b_{k, j} .
\end{aligned}
$$

The matrix $B=\left[b_{k, j}\right]$ is lower triangular, with diagonal entries $b_{k, k}=1$. Hence there is a well-defined inverse matrix $C=\left[c_{k, j}\right]$ which is also lower triangular. For each $2 \leqq n \leqq k$, let $\omega_{k}(n)$ be the integer defined by

$$
\omega_{k}(n)=\underset{n \leqq j \leqq k}{\operatorname{maximum}}\left\{\operatorname{order} c_{k, j} \text { in } \boldsymbol{Q} / \mathbb{Z}_{(p)}\right\} .
$$

Theorem 4.7. For each $2 \leqq n \leqq k$,

$$
E_{2}^{1,2 k+1}(S U(n)) \approx \omega_{k}(n) \boldsymbol{Z}_{(p)} / k ! \mathbb{Z}_{(p)} .
$$

Proof. From Section 2 (see also [2]), we know that $E_{2}^{*, *}(S U(n))$ may be calculated as the homology of the unstable cobar complex $C^{*, *}(M(n))$. Let $g_{k}$ be the element $-d\left(x_{2 k+1}\right)$ in $C^{1,2 k+1}(M(k+1))$; that is

$$
\begin{aligned}
g_{k} & =\psi\left(x_{2 k+1}\right)-1 \otimes x_{2 k+1} \\
& =\sum_{j=1}^{k-1}\left(\sum^{F^{*}} h_{s}\right)_{k-j}^{j} \otimes x_{2 j+1} \\
& =\sum_{j=1}^{k-1} \gamma_{k, j} \otimes x_{2 j+1}
\end{aligned}
$$

where $\gamma_{k, j}=\left(\sum^{F^{*}} h_{s}\right)_{k-j}^{j}$. The long exact sequence (4.1) shows that the homology class of $g_{k}$ generates $E_{2}^{1,2 k+1}(S U(k))$.

Next, let integers $\tau_{k}(n)$ for $1 \leqq n<k$ and rational numbers $a_{k, j}(n)$ for $1 \leqq n$ $\leqq k$, all $1 \leqq j$, be defined as follows. First, let $a_{k, j}(k)=\bar{e}\left(\gamma_{k, j}\right)$. Then recursively for $1 \leqq n<k$, let

$$
\tau_{k}(n)=\text { order of } a_{k, n}(n+1) \text { in } \boldsymbol{Q} / \boldsymbol{Z}_{(p)}
$$




$$
a_{k, j}(n)=\tau_{h}(n)\left(a_{k, j}(n+1)-a_{k, n}(n+1) a_{n, j}(n)\right) .
$$

We shall show by downward induction on $n$ that for $1 \leqq n \leqq k$, there are elements $g_{k}(n)$ in $U M(n)$ of the form

$$
g_{k}(n)=\sum_{j=1}^{n-1} \gamma_{k, j}(n) \otimes x_{2 j+1}
$$

where the $\gamma_{k, j}(n)$ are in $\Gamma$ with $\bar{e}\left(\gamma_{k, j}(n)\right)=a_{k, j}(n)$, and such that the homology class of $g_{k}(n)$ in $E_{2}^{1,2 k+1}(S U(n))$ represents the generator. This is true for $n=k$, because $g_{k}(k)=g_{k}$. Then assume inductively for $n<k$ that there is an element $g_{k}(n+1)$ as asserted. Consider the map

$$
E_{2}^{1,2 k+1}(S U(n+1)) \stackrel{\rho_{*}}{\longrightarrow} E_{2}^{1,2 k+1}\left(S^{2 n+1}\right) .
$$

Then $\rho_{*}\left(g_{k}(n+1)\right)=\gamma \otimes x_{2 n+1}$, where $\gamma$ is in $\Gamma$, with $\bar{e}(\gamma)=a_{k, n}(n+1)$. Then $\tau_{k}(n)=$ order of $\gamma \otimes x_{2 n+1}$ in $E_{2}^{1}, 2 k+1\left(S^{2 n+1}\right)$, so there is an element $a_{k}(n+1)$ in $A$, with

$$
d\left(a_{k}(n+1)\right)=\tau_{k}(n) \cdot \gamma \otimes x_{2 n+1} .
$$

Then the element

$$
\begin{aligned}
g_{k}(n) & =\tau_{k}(n) g_{k}(n+1)-d\left(a_{k}(n+1) x_{2 n+1}\right) \\
& =\sum_{j=1}^{n-1} \gamma_{k, j}(n) \otimes x_{2 j+1}
\end{aligned}
$$

generates $E_{2}^{1 \cdot 2 k+1}(S U(n))$, and satisifies

$$
\bar{e}\left(\gamma_{k, j}(n)\right)=a_{k, j}(n) .
$$

Next we define rational numbers $b_{k, j}(n)$ for all pairs of integers $(k, j)$ with $k \geqq n$ as follows. First, $b_{k, j}(k)=b_{k, j}$ as defined in (4.5). Then recursively for $n<k$, let

$$
b_{k, j}(n)=b_{k, j}(n+1)-b_{k, n}(n+1) b_{n, j} .
$$

Observe that the result of row-reducing the $k$-th row of the matrix $B=\left[b_{k, j}\right]$ by using rows $k-1, k-2, \ldots, n+1$ takes the $k$-th row

$$
\left\langle b_{k, 1}, b_{k, 2}, \ldots, b_{k, k-1}, 1,0, \ldots\right\rangle
$$

to the row

$$
\left\langle b_{k, 1}(n+1), \ldots, b_{k, n}(n+1), 0, \ldots, 0,1,0, \ldots\right\rangle .
$$

Hence, in the matrix $C$ (=the inverse of $B$ ), we have $c_{k, n}=-b_{k, n}(n+1)$. In particular, these two rational numbers have the same order in $\mathbb{Q} / \mathbb{Z}_{(p)}$. We 
assert that

$$
a_{k, j}(n)=\omega_{k}(n) \cdot b_{k, j}(n)
$$

which we shall show by downward induction on $n$. The statement is true for $n=k$, as $a_{k, j}(k)=b_{k, j}(k)$, and $\omega_{k}(k)=1$. Assume inductively that

$$
a_{k, j}(n+1)=\omega_{k}(n+1) b_{k, j}(n+1) .
$$

Then

$$
\begin{aligned}
a_{k, j}(n) & =\tau_{k}(n)\left(a_{k, j}(n+1)-a_{k, n}(n+1) a_{n, j}\right) \\
& =\tau_{k}(n) \omega_{k}(n+1)\left(b_{k, j}(n+1)-b_{k, n}(n+1) b_{n, j}\right) \\
& =\tau_{k}(n) \omega_{k}(n+1) b_{k, j}(n) \\
& =\operatorname{order}\left(a_{k, n}(n+1)\right) \cdot \omega_{k}(n+1) \cdot b_{k, j}(n) \\
& =\operatorname{order}\left(\omega_{k}(n+1) b_{k, n}(n+1)\right) \cdot \omega_{k}(n+1) \cdot b_{k, j}(n) \\
& =\max \left\{\omega_{k}(n+1), \operatorname{order}\left(b_{k, n}(n+1)\right\} b_{k, j}(n)\right. \\
& =\omega_{k}(n) b_{k, j}(n) .
\end{aligned}
$$

Finally, we consider the map

$$
\left(i_{n, k}\right)_{*}: E_{2}^{1,2 k+1}(S U(n)) \rightarrow E_{2}^{1,2 k+1}(S U(k))
$$

induced by the inclusion $i_{n, k}: S U(n) \rightarrow S U(k)$. By (4.2, (iv)), $\left(i_{n, k}\right)_{*}$ is a monomorphism, and by the above,

$$
\left(i_{n, k}\right)_{*} g_{k}(n)=\omega_{k}(n) \cdot g_{k} .
$$

Thus $E_{2}^{1,2 k+1}(S U(n)) \approx \omega_{k}(n) Z_{(p)} / k ! Z_{(p)}$ as asserted.

From these calculations, we can determine the kernel of the homomorphism

$$
E_{2}^{2, *}(S U(n)) \rightarrow E_{2}^{2}, *(S U(n+1)) .
$$

Recall from [2], that $E_{2}^{1,2 k+1}\left(S^{2 n+1}\right)$ is shown to be a cyclic group of order $\sigma_{k}(n)$ where $\sigma_{k}(n)$ is as follows. If $k-n \neq 0 \bmod 2 p-2, \sigma_{k}(n)=1$. Otherwise, write $k-n=(2 p-2) \cdot p^{m} \cdot q$, where $q$ is prime to $p$. Then for an odd prime $p$,

$$
\sigma_{k}(n)=\min \left\{p^{n}, p^{m+1}\right\}
$$

and for $p=2$,

$$
\sigma_{k}(n)=\left\{\begin{array}{l}
2, \quad \text { if } k-n \text { is odd }, \\
2, \quad \text { if } k=3, n=1, \\
4, \quad \text { if } k-n=2, n \geqq 2, \\
\min \left\{2^{n}, 2^{m+2}\right\}, \quad \text { otherwise }
\end{array}\right.
$$


Corollary 4.8. For $k<n$, the kernel of

$$
E_{2}^{2,2 k+1}(S U(n)) \rightarrow E_{2}^{2,2 k+1}(S U(n+1))
$$

is a cyclic group of order $\sigma_{k}(n) / \tau_{k}(n)$, where $\sigma_{k}(n)$ is as above, and $\tau_{k}(n)$ is the integer defined in the course of the proof of Theorem 4.7 .

Proof. From the long exact sequence (4.1), we must determine the cokernel of

$$
E_{2}^{1}, 2 k+1(S U(n+1)) \rightarrow E_{2}^{1,2 k+1}\left(S^{2 n+1}\right) .
$$

We have calculated that for $k<n$, the order of the image is $\tau_{k}(n)$. Therefore the cokernel has order $\sigma_{k}(n) / \tau_{k}(n)$ as asserted.

Q.E.D.

Remark 4.9. The results of Bousfield [3] concerning products in the unstable homotopy spectral sequence with coefficients in a ring may be generalized to a ring spectrum (in place of the ring). The statements of $[3,(8.2)]$ apply to our situation. In particular, the differentials are seen to act as derivations with respect to the action of $E_{r}^{*, *}\left(S^{2 n+1}\right)$ on $E_{r}^{*, *}(S U(n))$. The coboundary map

$$
\delta: E_{2}^{s, t}\left(S^{2 n+1}\right) \rightarrow E_{2}^{s+1, t}(S U(n))
$$

has the form $\delta(\alpha)= \pm \alpha \otimes d^{\prime}\left(x_{2 n+1}\right)$, for $\alpha$ in $E_{2}^{s, t}\left(S^{2 n+1}\right)$. Thus $\delta$ is map of spectral sequences. For an odd prime $p$, the elements of $E_{2}^{1,2 k+1}\left(S^{2 n+1}\right)$ are all permanent cycles [2], and their coboundaries are permanent cycles in $E_{2}^{2,2 k+1}(S U(n))$. When non-zero, these represent non-zero elements of $\pi_{*}(S U(n))$. A similar but more complicated statement holds for $p=2$, taking into account the differential $d^{3}$ on $E_{2}^{1}, *\left(S^{2 n+1}\right)$.

Examples 4.10. Some examples (of the upper left-hand corners) of the matrices of (4.5) are the following:

(1) For $p=2$,

$$
B=\left(\begin{array}{cccccc}
1 & 0 & 0 & 0 & 0 & 0 \\
\frac{1}{2} & 1 & 0 & 0 & 0 & 0 \\
0 & 1 & 1 & 0 & 0 & 0 \\
\frac{1}{4} & \frac{1}{4} & \frac{3}{2} & 1 & 0 & 0 \\
0 & \frac{1}{2} & \frac{3}{4} & 2 & 1 & 0 \\
0 & \frac{1}{4} & \frac{7}{8} & 3 & \frac{5}{2} & 1
\end{array}\right)
$$




$$
C=\left(\begin{array}{rrrrrr}
1 & 0 & 0 & 0 & 0 & 0 \\
-\frac{1}{2} & 1 & 0 & 0 & 0 & 0 \\
\frac{1}{2} & -1 & 1 & 0 & 0 & 0 \\
-\frac{7}{8} & \frac{5}{4} & -\frac{3}{2} & 1 & 0 & 0 \\
\frac{13}{8} & -\frac{9}{4} & -\frac{9}{4} & -2 & 1 & 0 \\
-\frac{15}{16} & \frac{5}{2} & \frac{1}{4} & \frac{5}{2} & -\frac{5}{2} & 1
\end{array}\right) .
$$

(2) For $p=3$,

$$
\begin{aligned}
B & =\left(\begin{array}{rrrrrr}
1 & 0 & 0 & 0 & 0 & 0 \\
0 & 1 & 0 & 0 & 0 & 0 \\
\frac{1}{3} & 0 & 1 & 0 & 0 & 0 \\
0 & \frac{2}{3} & 0 & 1 & 0 & 0 \\
0 & 0 & 1 & 0 & 1 & 0 \\
0 & \frac{1}{9} & 0 & \frac{4}{3} & 0 & 1
\end{array}\right) \\
C & =\left(\begin{array}{rrrrrr}
1 & 0 & 0 & 0 & 0 & 0 \\
0 & 1 & 0 & 0 & 0 & 0 \\
-\frac{1}{3} & 0 & 1 & 0 & 0 & 0 \\
0 & -\frac{2}{3} & 0 & 1 & 0 & 0 \\
\frac{1}{3} & 0 & -1 & 0 & 1 & 0 \\
0 & \frac{7}{9} & 0 & -\frac{4}{3} & 0 & 1
\end{array}\right) .
\end{aligned}
$$

(3) The methods of this section also apply to the unstable Adams-Novikov spectral sequence based on $M U$ (instead of $B P$ ). In this case, the definition of $\bar{e}$ becomes

$$
\begin{aligned}
& \bar{e}\left(m_{k}\right)=1 / k+1 \\
& \bar{e}\left(\eta\left(m_{k}\right)\right)=0
\end{aligned}
$$

where the $m_{k}=\left[C P^{k}\right] / k+1$ in $\pi_{2 k}(M U)$. The matrix $B$ of (4.5) is defined by 


$$
\left(Y+\frac{Y^{2}}{2}+\frac{Y^{3}}{3}+\cdots\right)^{j}=\sum_{k \geqq j} b_{k, j} Y^{k} .
$$

The matrix $C$ is the inverse of $B$, and then $\omega_{n}(k)$ is the least common multiple of the orders of $c_{k, j}$ in $\boldsymbol{Q} / \mathbb{Z}$ as $n \leqq j \leqq k$. The analogue to Theorem 4.7 becomes:

$$
E_{2}^{1,2 k+1}(S U(n)) \approx \omega_{k}(n) \mathbb{Z} / k ! \mathbb{Z}
$$

For this, the integral case,

$$
\begin{aligned}
& B=\left(\begin{array}{cccccc}
1 & 0 & 0 & 0 & 0 & 0 \\
\frac{1}{2} & 1 & 0 & 0 & 0 & 0 \\
\frac{1}{3} & 1 & 1 & 0 & 0 & 0 \\
1 & \frac{11}{12} & \frac{3}{2} & 1 & 0 & 0 \\
\frac{1}{1} & \frac{5}{6} & \frac{7}{4} & 2 & 1 & 0 \\
\frac{1}{6} & \frac{137}{180} & \frac{15}{8} & \frac{17}{6} & \frac{5}{2} & 1
\end{array}\right) \\
& C=\left(\begin{array}{cccccc}
1 & 0 & 0 & 0 & 0 & 0 \\
-\frac{1}{2} & 1 & 0 & 0 & 0 & 0 \\
\frac{1}{6} & -1 & 1 & 0 & 0 & 0 \\
-\frac{1}{24} & \frac{7}{12} & -\frac{3}{2} & 1 & 0 & 0 \\
\frac{1}{120} & -\frac{1}{4} & \frac{5}{4} & -2 & 1 & 0 \\
-\frac{1}{720} & \frac{31}{360} & -\frac{3}{4} & \frac{13}{6} & -\frac{5}{2} & 1
\end{array}\right) .
\end{aligned}
$$

\section{§5. Some Calculations in $\mathbb{E}_{2}^{*, *}\left(S^{2 n+1}\right)$}

In this section, we describe a method of making resolutions of unstable $\Gamma$-comodules which are convenient for calculations. We use these resolutions to establish vanishing lines for unstable Ext groups. In particular, we give a vanishing line for $E_{2}^{*}$,* $\left(S^{2 n+1}\right)$. We also make some calculations of $E_{2}^{2}, *\left(S^{2 n+1}\right)$ in low stems, which together with the calculations of $E_{2}^{1}, *\left(S^{2 n+1}\right)$ of [2], give some of the unstable groups $\pi_{*}\left(S^{2 n+1}\right)$. 
Throughout this section, a prime $p$ is fixed, $B P$ is the Brown-Peterson spectrum associated with $p, \pi_{*}(B P)=A$, and $\pi_{*}(B P \wedge B P)=\Gamma$. The category of connected $A$-modules is called $\mathscr{A}$. The category $\mathscr{U}$ of unstable $\Gamma$-comodules is defined in Section 2 by the cotriple $\left(U, \varepsilon^{U}, \delta^{U}\right)$ on $\mathscr{A}$. Let $J: \mathscr{U} \rightarrow \mathscr{A}$ be the forgetful functor. Then for $M$ in $\mathscr{A}$ and $N$ in $\mathscr{U}$, there are natural isomorphisms $\alpha$ and $\beta$ :

$$
\operatorname{Hom}_{\mathscr{U}}(N, U(M)) \underset{\beta}{\stackrel{\alpha}{\rightleftarrows}} \operatorname{Hom}_{\mathscr{A}}(J(N), M) \text {. }
$$

If $f: N \rightarrow U(M)$ is a map in $\mathscr{U}$, then $\alpha(f)=\varepsilon^{U} \circ f: J(N) \rightarrow M$ is a map in $\mathscr{A}$. If $g: J(N) \rightarrow M$ is a map in $\mathscr{A}$, then $\beta(g)=U(g) \circ \psi: N \rightarrow U(M)$ is a map in $\mathscr{U}$. Specifically, if $x$ is in $N$, with $\psi(x)=\sum_{i} \gamma_{i} \otimes x_{i}$, then

$$
\beta(g)(x)=\sum_{i} \gamma_{i} \otimes g\left(x_{i}\right) .
$$

In particular, a map $f: N \rightarrow U(M)$ in $\mathscr{U}$ is determined by the map $g=\varepsilon^{U} \circ f$ by formula (5.2).

Recall that $A[t]$ is the free $A$-module on one generator $\iota_{t}$ of degree $t$, with trivial $\Gamma$-coaction. Then for any $A$-module $M$,

$$
\operatorname{Hom}_{\mathscr{U}}(A[t]) \approx \operatorname{Hom}_{\mathscr{A}}(J(A[t]), M) \approx M_{t} .
$$

Acyclic Resolutions (5.3). Suppose that $M$ is in $\mathscr{U}$. Then an acyclic resolution of $M$ by models is a sequence

$$
0 \rightarrow M \stackrel{\partial_{-1}}{\longrightarrow} U\left(M^{0}\right) \stackrel{\partial_{0}}{\longrightarrow} U\left(M^{1}\right) \stackrel{\partial_{1}}{\longrightarrow} U\left(M^{2}\right) \rightarrow \cdots
$$

which is acyclic, and the maps $\partial_{-1}, \partial_{0}, \partial_{1}, \ldots$ are in $\mathscr{U}$. From this, we obtain a complex

$$
M^{0} \stackrel{d^{0}}{\longrightarrow} M^{1} \stackrel{d^{1}}{\longrightarrow} M^{2} \rightarrow \cdots
$$

where, for each $i \geqq 0, d$ is the map

$$
d^{i}(x)=\varepsilon^{U} \circ \partial_{i}(1 \otimes x) .
$$

Then, by standard homological algebra in the abelian category $\mathscr{U}$, we have:

$$
\operatorname{Ext}_{\mathscr{U}}^{s, t}(M) \approx\left(\operatorname{ker} d^{s} / \mathrm{im} d^{s-1}\right)_{t} .
$$

Our next step is to find convenient resolutions for certain kinds of modules.

Definition 5.5. An $A$-module $M$ is called quasi-free if $M \approx F / R$ where $F$ is a free $A$-module with homogeneous basis $\left\{x_{\alpha}\right\}$, and $R$ is a sub- $A$-module of $F$ which is generated by homogeneous elements $\{r\}$, each of which is of the form 


$$
r=\sum_{\alpha} c_{\alpha} x_{\alpha}
$$

where the $c_{\alpha}$ are in $\mathbb{Z}_{(p)}$.

The usefulness of quasi-free modules is the following.

Proposition 5.6. Let $M$ be a quasi-free A-module. Let $n$ be a fixed positive integer, and let $N$ be the sub-A-module of $M$ spanned by the elements of $M_{m}$ for $m<n$. Then

$$
M \approx N \oplus M / N
$$

as A-modules.

Proof. Write $M \approx F / R$ as given in the definition (5.5). Then let

$$
\begin{aligned}
& F_{0}=\operatorname{span}\left\{x_{\alpha} \mid \text { degree }\left(x_{\alpha}\right)<n\right\} \\
& F_{1}=\operatorname{span}\left\{x_{\alpha} \mid \text { degree }\left(x_{\alpha}\right) \geqq n\right\} \\
& R_{0}=\operatorname{span}\{r \mid \text { degree }(r)<n\} \\
& R_{1}=\operatorname{span}\{r \mid \text { degree }(r) \geqq n\} .
\end{aligned}
$$

Then it is immediate that $F=F_{0} \oplus F_{1}, R=R_{0} \oplus R_{1}$, and $N \approx F_{0} / R_{0}, M / N$ $\approx F_{1} / R_{1}$. Thus

$$
\begin{aligned}
M & \approx F_{0} / R_{0} \oplus F_{1} / R_{1} \\
& \approx N \oplus M / N .
\end{aligned}
$$

Proposition 5.7. Let $M$ be an unstable $\Gamma$-comodule, which is quasi-free as an $A$-module, and suppose that connectivity $(M)=k-1$, with $k \geqq 2 p-1$. Then

$$
\begin{array}{lll}
\operatorname{Ext}_{\mathscr{U}}^{2 s, t}(M)=0, & \text { for } & t<2(p-1) p s+k, \\
\operatorname{Ext}_{\mathscr{K}}^{2 s+1, t}(M)=0, & \text { for } & t<2(p-1)(p s+1)+k
\end{array}
$$

Proof. By downward induction on the connectivity of $M$. If $M$ is highly connected, (5.7) holds by the Miller-Zahler vanishing line for stable $\Gamma$-comodules ([6], [12]). Assume inductively that (5.7) holds for connectivity $\geqq k$ and let $M$ be an unstable $\Gamma$-comodule, quasi-free as an $A$-module, with connectivity ( $M$ ) $=k-1$, and $k \geqq 2 p-1$. We construct an acyclic resolution of $M$ by models as follows.

Let $S^{2 p-2} M$ be the $\left(S^{2 p-2} M\right)_{2 p-2+n}=M_{n}$. For $x$ in $M$, let $S^{2 p-2} x$ denote the corresponding element in $S^{2 p-2} M$. The unstable $\Gamma$-comodule structure on $M$ is a map $\psi: M \rightarrow U(M)$. Recall from (2.13) that $U(M)$ is spanned by 
the elements $h^{I} \otimes x$ where $x$ is in $M$, and $2 l(I)<$ degree $(x)$. Thus coker $\psi$ is spanned by the same $h^{I} \otimes x$ but excluding the $1 \otimes x$. We define an $A$-linear map

$$
f: \operatorname{coker} \psi \rightarrow S^{2 p-2} M
$$

by

$$
\begin{aligned}
& f\left(h_{1} \otimes x\right)=S^{2 p-2} x \\
& f\left(h^{I} \otimes x\right)=0, \text { for } h^{I} \neq h_{1} .
\end{aligned}
$$

It is easy to see that $f$ is well defined.

Let $N$ be the sub- $A$-module of coker $\psi$ spanned by the elements of degree strictly less than $2(p-1) p+k$. Then by (5.6),

$$
\operatorname{coker} \psi \approx N \oplus(\operatorname{coker} \psi / N) \text {. }
$$

Let $M^{1}=S^{2 p-2} M \oplus\left(\operatorname{coker} \psi_{i}^{\prime} N\right)$, and let

$$
(f \oplus \lambda): \operatorname{coker} \psi \rightarrow M^{1}
$$

be the map where $f$ is as above and $\lambda$ is the natural projection. Then $g=$ $\beta(f \oplus \lambda)$ is a map

$$
g: \operatorname{coker} \psi \rightarrow U\left(M^{1}\right) .
$$

We claim that (i): $g$ is one-one; and (ii): $g$ is subjective in degrees $<2(p-1) p$ $+k$. Note that $M$ quasi-free (as an $A$-module) implies that $U(M)$ and coker $\psi$ are quasi-free also. Consider

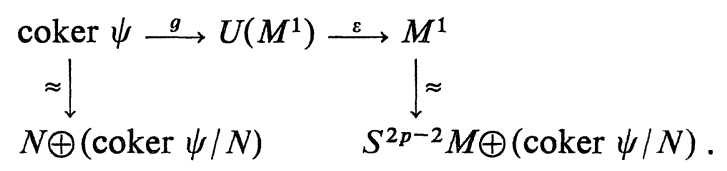

Suppose that $x_{1}$ is in $N$ and $x_{2}$ is in $(\operatorname{coker} \psi / N)$ and that $g\left(x_{1} \oplus x_{2}\right)=0$. Then the projection of $\varepsilon g\left(x_{1} \oplus x_{2}\right)$ on coker $\psi / N$ is $x_{2}$, so $x_{2}=0 . \quad N$ is spanned by elements of the form $h^{I} \otimes x$ which have degree $<2(p-1) p+k$. Thus $N$ is spanned by elements $h_{1}^{n} \otimes x$, where $0<n<p$. Then

$$
g\left(h_{1}^{n} \otimes x\right)=\left(n h_{1}^{n-1} \otimes S^{2 p-2} x\right)+h_{1}^{n} \otimes \varepsilon g(1 \otimes x) .
$$

Statements (i) and (ii) now follow readily by induction on $n$.

We next construct an acyclic resolution of $M$ by models

$$
0 \rightarrow M \stackrel{\hat{c}_{-1}}{\longrightarrow} U(M) \stackrel{\hat{\partial}_{0}}{\longrightarrow} U\left(M^{1}\right) \stackrel{\hat{\partial}_{1}}{\longrightarrow} U\left(M^{2}\right) \longrightarrow \cdots
$$


as follows. The map $\partial_{-1}=\psi$, the coaction map for $M$. The map $\partial_{0}$ is the composite

$$
U(M) \stackrel{\lambda}{\longrightarrow} \operatorname{coker} \psi \stackrel{g}{\longrightarrow} U\left(M^{1}\right)
$$

where $\lambda$ is the natural quotient map and $g$ is defined above. Let $M^{2}=\operatorname{coker} \partial_{0}$, and for $i>2$, let $M^{i}=U^{i-2}\left(M^{2}\right)$. For $i \geqq 1$, the $\partial_{i}$ are the maps in the cobar resolution for $M^{2}$. Then by (5.4),

$$
\operatorname{Ext}_{\mathscr{\mathcal { U }}}^{s, t}(M) \approx\left(\operatorname{ker} d^{s} / \operatorname{im} d^{s-1}\right)_{t}
$$

of the sequence

$$
M \stackrel{d^{0}}{\longrightarrow} M^{1} \stackrel{d^{1}}{\longrightarrow} M^{2} \stackrel{d^{2}}{\longrightarrow} \cdots .
$$

By construction, $M_{t}^{1}=0$, for $t<2(p-1)+k$ and $M_{t}^{2}=0$, for $t<2(p-1)+k$. Therefore

$$
\begin{aligned}
& \operatorname{Ext}_{\mathscr{U}}^{1 \cdot t}(M)=0, \quad \text { for } t<2(p-1)+k, \\
& \operatorname{Ext}_{\mathscr{U}}^{2, t}(M)=0, \text { for } t<2(p-1) p+k .
\end{aligned}
$$

For $s \geqq 3$, we have isomorphisms

$$
\operatorname{Ext}_{\mathscr{U}}^{s, t}(M) \approx \operatorname{Ext}_{\mathscr{U}}^{s-2, t}\left(M^{2}\right) .
$$

The statement of (5.7) follows by induction.

Q.E.D.

Corollary 5.8. For $n \geqq p-1$,

$$
\begin{array}{lll}
E_{2}^{2 s, t}\left(S^{2 n+1}\right)=0, & \text { for } & t<2(p-1) p s+2 n+1, \\
E_{2}^{2 s+1, t}\left(S^{2 n+1}\right)=0, & \text { for } & t<2(p-1)(p s+1)+2 n+1 .
\end{array}
$$

Definition 5.9. For each pair of positive integers $(m, k)$ with $m<p$, let $M_{m}(k)$ be the free $A$-module with generators

$$
\left\{x_{2 m+1+2(p-1) i}\right\}, \quad 0 \leqq i<k .
$$

The $\Gamma$-coaction on $M_{m}(k)$ is given by formula (3.4). (The coaction is defined because $\Gamma$ is sparse, i.e. $\Gamma_{t}=0$ if $t \not \equiv 0 \bmod (2 p-2)$.)

Corollary 5.10.

$$
\begin{array}{lll}
\operatorname{Ext}_{\mathscr{U}}^{2 s+1, t}\left(M_{m}(k)\right)=0, & \text { for } & t<(2 p-2)(p s+k)+2 m+1, \\
\operatorname{Ext}_{\mathscr{Z}}^{2 s+2, t}\left(M_{m}(k)\right)=0, & \text { for } & t<2(p-1)(p s+k+1)+2 m+1 .
\end{array}
$$

Proof. The short exact sequence of unstable $\Gamma$-comodules

$$
0 \rightarrow M_{m}(k) \rightarrow M_{m}(k+1) \rightarrow A[2 m+1+2(p-1) k] \rightarrow 0
$$


induces a long exact sequence of Ext groups. Then (5.10) follows by induction, using (3.3) and (5.8).

Q.E.D.

Corollary 5.11. Let $n \leqq p-1$. Then for each $s \geqq 0$,

$$
\begin{array}{lll}
E_{2}^{2 s+1, t}\left(S^{2 n+1}\right)=0, & \text { for } & t<2(p-1)(p s+1)+2 n+1, \\
E_{2}^{2 s+2, t}\left(S^{2 n+1}\right)=0, & \text { for } & t<2(p-1)(p s+2)+2 n+1 .
\end{array}
$$

Proof. This is the statement of (5.10) with $k=1$.

Q.E.D.

Remarks 5.12. (i) In [7], spaces $B_{m}(k)$ are constructed, with

$$
P H_{*}\left(B_{m}(k) ; B P\right) \approx M_{m}(k) .
$$

(In [7], $B_{m}(k)$ is denoted $B_{m}^{k}(p)$.) As a consequence of $[7,(3.5)]$, the unstable Adams-Novikov spectral sequence for $S U(n)$ breaks up into a direct sum of $(p-1)$ spectral sequences, with

$$
E_{2}(S U(n)) \approx \underset{1 \leqq m<p}{\oplus} \operatorname{Ext}_{\mathscr{L}}\left(M_{m}(k(n, m))\right)
$$

where $k(n, m)=\left[\frac{n-m-1}{p}\right]+1$.

(ii) The vanishing line (5.11) cannot be improved on $S^{3}$ as $\beta_{1}^{r} \alpha_{1}^{2} \neq 0$ in $E_{2}\left(S^{3}\right)$ for all $r$. $\quad\left(\beta_{1}\right.$ and $\alpha_{1}$ denote the classes in $E_{2}$ which represent the corresponding homotopy elements.) This is not the best possible for higher spheres; see Theorem 5.16, below.

Corollary 5.13. For $t-s<2(p-1)(p(p-1)+k)+2 m-1$,

$$
\operatorname{Ext}_{\mathscr{L}}^{s, t}\left(M_{m}(k)\right)=E_{\infty}^{s, t}\left(B_{m}(k)\right)
$$

and there are no extensions if $t-s<2(p-1)(p(p-1)+k-1)+2 m$.

Proof. Sparseness.

Q.E.D.

Example 5.14. If $n<p-1$, and $q<2 p(p-1)+2 n+1$,

$$
\pi_{q}(S U(n)) \approx E_{2}(S U(n))
$$

and all non-zero elements occur in filtrations 0,1 and 2 . Compare with [12; (5.7.4)].

Remarks 5.15. The above applies to any space $X$ such that $H_{*}(X ; B P)$ is free as an $A$-module and cofree as a coalgebra, and such that $P H_{*}(X ; B P)$ as an unstable $\Gamma$-comodule is isomorphic to a direct sum of $M_{m}(k)$ 's. In particular, for $p$ an odd prime, by $[7 ;(4.1)]$, these methods apply to $\operatorname{Spin}(n)$ and to $S p(n)$. 
We leave the details to the reader.

To illustrate the method (5.7) of resolving unstable $\Gamma$-comodules, we show the following (see $[9 ;(7.1)]$ ).

Theorem 5.16. Let a prime $p$ be fixed. For $q$ in the range $2 n+1<q$ $<2 p^{2}+2 n-4$, the p-localized group $\pi_{q}\left(S^{2 n+1}\right)$ is zero except in the following cases:

(i) For $0<k<p$,

$$
\pi_{(2 p-2) k+2 n}\left(S^{2 n+1}\right) \approx \mathbb{Z}_{p}
$$

Also (the case when $k=p$ ),

$$
\begin{aligned}
\pi_{(2 p-2) p+2 n}\left(S^{2 n+1}\right) & \approx \mathbb{Z}_{p}, \quad \text { for } n=1, \\
& \approx \mathbb{Z}_{p^{2}}, \quad \text { for } n>1 .
\end{aligned}
$$

(ii) For $n<k<p$

$$
\pi_{(2 p-2) k+2 n-1}\left(S^{2 n+1}\right) \approx \mathbb{Z}_{p} .
$$

Also (the case when $k=p$ ),

$$
\begin{aligned}
\pi_{(2 p-2) p+2 n-1}\left(S^{2 n+1}\right) & \approx \mathbb{Z}_{p}, \quad \text { for } n=1, \text { or } n \geqq p, \\
& \approx \mathbb{Z}_{p^{2}}, \quad \text { for } 1<n<p .
\end{aligned}
$$

Proof. We prove (5.16) for $n<p$. The proof for $n \geqq p$ is similar. For the range $2 n+1<t-s<2 p^{2}+2 n-4$, by (5.11), we have $E_{2}^{s, t}\left(S^{2 n+1}\right)=0$ for $s>2$. Hence in this range,

$$
\begin{array}{rlrl}
\pi_{q}\left(S^{2 n+1}\right) \approx E_{2}^{1, q+1}\left(S^{2 n+1}\right), & \text { for } q \text { even }, \\
& \approx E_{2}^{2, q+2}\left(S^{2 n+1}\right), & & \text { for } q \text { odd }
\end{array}
$$

We shall calculate $E_{2}^{* * *}\left(S^{2 n+1}\right)$ in this range by an acyclic resolution of $A[2 n+1]$ as follows. Let $M^{0}$ be the free $A$-module with one generator $x$ of degree $2 n+1$. Let $M^{1}$ be the free $A$-module with one generator $y$ of degree $(2 p-2)+2 n+1$. Let $M^{2}$ be the $A$-module generated by elements $z_{1}, z_{2}, \ldots$, $z_{p-n}$, where degree $\left(z_{i}\right)=(2 p-2)(n+i)+2 n+1$, modulo the ideal of relations:

$$
\begin{gathered}
p z_{1} \\
(n+k) v_{1} z_{k}-p(n+k+1) z_{k+1} .
\end{gathered}
$$

We note that these relations imply that in $M^{2}$,

$$
p^{i} z_{i}=0, \quad \text { for } 1 \leqq i<p-n,
$$




$$
p^{p-n+1} z_{p-n}=0
$$

Let $M^{3}$ be the sub- $A$-module of $\Gamma \otimes_{A} M^{2}$ generated by the elements $\left\{h_{1}^{k} \otimes z_{i}\right\}$, where $1 \leqq i \leqq p-n$ and $k>0$, and with relations induced from those in $M^{2}$. Let

$$
\partial_{-1}: A[2 n+1] \rightarrow M^{0}
$$

be the map with $\partial_{-1}\left(\iota_{2 n+1}\right)=1 \otimes x$. By $[2, \S 8]$, in the range of dimensions under consideration, $U\left(M^{0}\right)$ has an $A$-basis consisting of the elements:

$$
\begin{array}{ll}
h_{1}^{k} \otimes x, & \text { for } \quad 0 \leqq k \leqq n, \\
h_{1}^{k} v_{1} \otimes x, & \text { for } \quad n \leqq k<p-1 .
\end{array}
$$

Let $\partial_{0}: U\left(M^{0}\right) \rightarrow U\left(M^{1}\right)$ be the $A$-linear map defined by

$$
\begin{aligned}
& \partial_{0}\left(h_{1}^{k} \otimes x\right)=k h_{1}^{k-1} \otimes y^{\prime}, \quad \text { for } 0 \leqq k \leqq n, \\
& \partial_{0}\left(h_{1}^{k} v_{1} \otimes x\right)=k v_{1} h_{1}^{k-1} \otimes y-p(k+1) h_{1}^{k} \otimes y, \quad \text { for } n \leqq k<p-1
\end{aligned}
$$

Let $\partial_{1}: U\left(M^{1}\right) \rightarrow U\left(M^{2}\right)$ be the $A$-linear map defined by

$$
\begin{aligned}
& \partial_{1}\left(h_{1}^{k} \otimes y\right)=0, \quad \text { for } 0 \leqq k<n, \\
& \partial_{1}\left(h_{1}^{k} \otimes y\right)=\sum_{i=0}^{k-n}\left(\begin{array}{c}
k \\
i
\end{array}\right) h_{1}^{i} \otimes z_{k-n-i+1}, \quad \text { for } \quad n \leqq k<p-1 .
\end{aligned}
$$

Finally, let $\partial_{2}: U\left(M^{2}\right) \rightarrow U\left(M^{3}\right)$ be the composite

$$
U\left(M^{2}\right) \stackrel{\lambda}{\longrightarrow} U\left(M^{2}\right) / \operatorname{Im} \partial_{1} \approx M^{3} \stackrel{\psi}{\longrightarrow} U\left(M^{3}\right)
$$

where $\lambda$ is the natural quotient map and $\psi$ is the coaction map for $M^{3}$.

It is readily verified that

$$
0 \rightarrow A[2 n+1] \stackrel{\hat{\hat{c}-1}}{\longrightarrow} U\left(M^{0}\right) \stackrel{\hat{o}_{0}}{\longrightarrow} U\left(M^{1}\right) \stackrel{\hat{o}_{1}}{\longrightarrow} U\left(M^{2}\right) \stackrel{\hat{\partial}_{2}}{\longrightarrow} U\left(M^{3}\right)
$$

is an acyclic resolution of $A[2 n+1]$ in the range of dimensions under consideration. Thus, by (5.4), in the range $t-s<2 p^{2}+2 n-4, E_{2}^{s, t}\left(S^{2 n+1}\right)$ may be calculated as the homology of the complex

$$
M^{0} \stackrel{d^{0}}{\longrightarrow} M^{1} \stackrel{d^{1}}{\longrightarrow} M^{2} \stackrel{d^{2}}{\longrightarrow} M^{3} .
$$

The maps $d^{i}$ are given in (5.3), which in this case become:

$$
\begin{aligned}
& d^{0}\left(v_{1}^{k} x\right)=p k v_{1}^{k-1} x \\
& d^{1}\left(v_{1}^{k} y\right)= \begin{cases}0, & \text { if } n>1, \\
0, & \text { if } n=1, k<p-1, \\
p^{p-1} z_{p-1}, & \text { if } n=1, k=p-1 .\end{cases}
\end{aligned}
$$


Furthermore, the kernel of $d^{2}$ is generated by the elements $\left\{v_{1}^{m} p^{k-1} z_{k}\right\}$ for $m \geqq 0, k \geqq 1$. The relations in $M^{3}$ imply that

$$
\begin{aligned}
& v_{1}^{k} z_{1}=p^{k} z_{k+1}, \quad \text { for } \quad k<p-n-1, \\
& v_{1}^{p-n-1} z_{1}=p^{p-n} z_{p-n}
\end{aligned}
$$

(to within a factor of a unit in $\mathbb{Z}_{(p)}$ ). Thus in the range $t<2 p^{2}+2 n-3$, $E_{2}^{1, t}\left(S^{2 n+1}\right)$ is generated by the classes $\left\{v_{1}^{k} y\right\}$ (except when $n=1$, and $k=p-1$, in which case $p v_{1}^{p-1} y$ is the generator). These classes have the orders asserted in $\left(5.14\right.$, (i)). Also, in the range $t<2 p^{2}+2 n-2, E_{2}^{2, t}\left(S^{2 n+1}\right)$ is generated by the classes $\left\{v_{1}^{k} z_{1}\right\}$ for $0 \leqq k<p-n-1$, and by $v_{1}^{p-n-1} z_{1} / p$. These classes have the orders asserted in (5.16), (ii).

Q.E.D.

Remarks 5.17. In the range $q<2 p^{2}-5$, the double suspension homomorphism

$$
\pi_{2 n+1+q}\left(S^{2 n+1}\right) \rightarrow \pi_{2 n+3+q}\left(S^{2 n+3}\right)
$$

is the multiplication by $p$ except in the following cases, when the double suspension sends a generator to a generator.

(1) $q=(2 p-2) k-1$ and $0<k<p$, all $n$, $q=(2 p-2) p-1$ and $n>1$.

(2) $q=(2 p-2) k-2$, and $n \geqq p-1$.

\section{References}

[1] Adams, J. F., Stable homotopy and generalized homology, University of Chicago Press, 1974.

[2] Bendersky, M., Curtis E. B. and Miller, H. R., The unstable Adams spectral sequence for generalized homology, Topology, 17 (1978), 229-248.

[3] Bousfield A. K. and Kan, D. M., Products and pairings in the homotopy spectral sequence, Trans. Amer. Math. Soc., 177 (1963), 319-343.

[4] Curtis, E. B., Simplicial homotopy theory, Advances in Mlath., 6 (1971), 107-209.

[5] Hazewinkel, M., A universal formal group and complex cobordism, Bull. Amer. Math. Soc., 81 (1975), 930-933.

[6] Miller, H. R., Some algebraic aspects of the Adams-Novikov spectral sequence, Thesis, Princeton University, 1974.

[7] Mimura, M., Nishida G. and Toda, H., Mod- $p$ decomposition of compact Lie groups, Publ, RIMS, Kyoto Univ., 13 (1977), 627-680.

[ 8 ] Toda, H., A topological proof of theorems of Bott, Borel and Hirzebruch, Ment. Kyoto Univ., 32 (1958), 103-119.

[9] —, On iterated suspension I, J. Math. Kyoto Univ., 5 (1965), 87-142.

[10] Wilson, W. S., The $\Omega$-spectrum for Brown-Peterson cohomology, Part II, Amer. 
J. of Math., 97 (1975), 101-123.

[11] Zabrodsky, A., Hopf spaces, North-Holland Math. Studies 22, North-Holland Publ. Co., Amsterdam, 1976.

[12] Zahler, R. S., The Adams-Novikov spectral sequence for the spheres, Ann. of Math., 96 (1972), 480-504.

Note added in proof: In order to compute the matrix $C$ in Section 4 it is convenient to use the following observation: For a polynomial $A(Y)=Y+a_{2} Y^{2}+\cdots$ let $[A(Y)]$ be the matrix with the entries in the $j$-th column given by the coefficients of $(A(Y))^{j}$. Then $[B(Y)]$ $[A(Y)]=[A B(Y)]$. 